\title{
Psychotropics and hyponatraemia
}

\author{
Robyn McAskill and David Taylor
}

Hyponatraemia is usually defined as a serum sodium concentration below $135 \mathrm{mmol} / \mathrm{l}$. The condition is rarely symptomatic until serum sodium falls below $120 \mathrm{mmol} / 1$ and symptoms are more usually associated with values around $110 \mathrm{mmol} / 1$. These symptoms include confusion and restlessness, progressing to drowsiness, myoclonic jerks, generalised convulsions and eventually coma. In a general hospital population about $1 \%$ of patients develop hyponatraemia (Rutsky, 1992), whereas in psychiatric patients the prevalence has been reported to range from $3.3 \%$ to $12.2 \%$ (Ohsawa et al, 1992).

The syndrome of inappropriate antidiuretic hormone secretion (SIADH) is the most important mechanism underlying drug-induced hyponatraemia. It was first described by Schwartz and colleagues in 1957 as a manifestation of dilutional hyponatraemia caused by water retention in euvolaemic patients with normal renal, liver, adrenal and thyroid function. SIADH can be diagnosed by finding a concentrated urine (sodium $>20 \mathrm{mmol} / \mathrm{l}$ ) in the presence of hyponatraemia or low plasma osmolality ( $<260 \mathrm{mmol} / \mathrm{kg}$ ) and an absence of hypovolaemia. Drugs are probably the most common cause of SLADH: opiates, chlorpropamide, cytotoxics and psychotropics are most often implicated. A wide range of psychotropic drugs have been associated with the development of hyponatraemia but the majority of reports are of single cases. This may indicate that hyponatraemia is idiosyncratic in nature and it certainly makes very difficult any accurate estimate of incidence, comparative or otherwise.

Estimates of the incidence of hyponatraemia with psychotropics are also confounded by other factors. Dubovsky et al (1973), reported that psychosis itself can cause hyponatraemia independent of drug therapy. Risk factors for the development of hyponatraemia in psychiatric patients are said to include old age, history of prior hyponatraemia, polydipsia and schizophrenia (Thomas \& Verbalis, 1995), smoking, an early onset of the psychiatric disorder $(<20$ years), longer duration of psychiatric disease ( $>10$ years) and prolonged admission (Ohsawa et al, 1992). Nevertheless, it is accepted that psychotropics are an important cause of hyponatraemia in the mentally ill.

Antiepileptics. Carbamazepine was one of the first drugs to be implicated in causing hyponatraemia (through SIADH) and this is often seen in clinical practice. The number of reports assoclated with the other antiepileptic drugs listed is comparatively low.

Antidepressants. All classes of antidepressants have been associated with hyponatraemia. The majority of reports with tricyclics and SSRIs have occurred in the elderly. While all SSRIs have been implicated (e.g. Vishwanath et al, 1991; Goddard \& Paton, 1992: Thornton \& Resch, 1995), there are few reports of hyponatraemia associated with MAOIs. The British National Formulary now outlines the recent CSM advice on hyponatraemia and antidepressants (Committee on Safety of Medicines, 1994).

Antipsychotics. Most classes of antipsychotics have been associated with hyponatraemia, although infrequently. Glusac et al (1990), reported a patient developing hyponatraemia with thioridazine, haloperidol, fluphenazine, thiothixene and trifluoperazine in sequence, but not molindone (an antipsychotic used in the USA). Oglivie \& Croy (1992), reported one patient developing hyponatraemia with clozapine. Conversely, there have been reports of clozapine decreasing polydipsia and improving hyponatraemia in patients on other neuroleptics (Wakefield \& Colls, 1996; Leadbetter \& Shutty, 1994). Clozapine can cause urinary frequency and may actually antagonise ADH.

Anxiolytics and hypnotics. There are few reports of hyponatraemia associated with anxiolytics and hypnotics.

Psychotropic-induced SIADH may be expected to manifest itself within a few days of starting a new drug. Occasionally, it may occur much later (Thomas \& Verbalis, 1995). Treatment of hyponatraemia involves identification of the underlying 
cause, discontinuing the offending drug and correcting volume abnormalities. Sodium levels should be checked in all psychiatric patients on admission. All those at risk of hyponatraemia (e.g. elderly taking antidepressants) should have frequent sodium level determinations. Hyponatraemia should also be suspected whenever patients receiving psychotropic drugs show marked changes in their underlying disease, significant increases in bodyweight, seizures or other symptoms of hyponatraemia (most commonly confusion).

Immediate management of hyponatraemia is dependent upon its severity and duration and whether neurological symptoms are present. SIADH is usually treated by fluid restriction unless dehydration is suspected. In hyponatraemia with dehydration, normal saline infusion should be used. Patients with neurological signs such as confusion, vomiting and convulsions require more aggressive treatment, usually with hypertonic saline. Severe cases such as this should be managed by acute medical services.

The majority of patients with hyponatraemia will respond to cessation of psychotropic drugs and/or one of the above therapies. If long-term fluid restriction proves to be intolerable, and the offending drug cannot be withdrawn, demeclocycline, a drug that inhibits $\mathrm{ADH}$ at the kidneys. may be used to treat SIADH. The usual dose is 600-1200 mg daily in divided doses. Demeclocycline may cause photosensitivity reactions.

Some patients seem especially prone to psychotropic-induced hyponatraemia. However, prophylactic use of demeclocycline in polydipsic patients has not proved successful (Alexander et al, 1991). Other prophylactic treatments including naloxone, captopril, propranolol, phenytoin and electrolyte containing drinks have also proved unsuccessful (Thomas \& Verbalis, 1995).

Once psychotropic-induced hyponatraemia has occurred, future treatment is made difficult. There are several options available.

\section{Rechallenge with the same drug}

Drug rechallenge will help to give more accurate information about the true incidence of hyponatraemia related to specific psychotropics. However, there are few case reports in the literature, probably because drug rechallenge is considered inappropriate and potentially dangerous. Staab et al (1990) describe a patient developing hyponatraemia with fluoxetine but not on rechallenge. However, Thornton \& Resch (1995) reported a recurrence of hyponatraemia with sertraline on rechallenge in one patient. Rechallenge should not therefore be attempted unless there is a pressing need to establish the cause of hyponatraemia.
Swap to a different drug of the same class

There have been single case reports of patients developing SIADH with more than one SSRI. Flint et al (1996), reported recurrent hyponatraemia with separate trials of fluoxetine and paroxetine and Jackson et al (1995) reported recurrent hyponatraemia with fluoxetine and sertraline. Based on this limited evidence, one SSRI should not usually be swapped for another. There is little information on swapping within other classes of drugs.

\section{Swap to a different drug class}

We are not aware of any reports describing the effect of swapping from one class of antidepressant to another. However, there are several case reports in the literature regarding swapping neuroleptic classes. These cases have been discussed above.

\section{ECT}

This may be a suitable option in some circumstances.

\section{Conclusion}

Hyponatraemia is a potentially dangerous condition occurring frequently in both psychiatric illness and in patients taking psychotropic drugs. Careful monitoring is therefore essential to minimise the risk to patients. All psychotropic drugs should be assumed to have the potential to cause hyponatraemia. Mild hyponatraemia may be treated in psychiatric institutions but more serious cases should be referred. When psychotropic-induced hyponatraemia does occur, the offending drug should be withdrawn and a drug of a different class cautiously substituted.

\section{References}

ALEXANDER, R. C. KARP, B. I. THOMPSON, S. et al (1991) A double blind placebo-controlled trial of demeclocycline treatment of polydipsia-hyponatraemia in chronically psychotic patients. Biological Psychiatry. 30, 417-420.

COMMITTEE ON SAFETY OF MEDICINES (1994) Antidepressantinduced hyponatraemia. Current Problems in Pharmacovigilance, 20, 5-6.

Dubovsky, S. L., Grabon, S., BERL. T., et al (1973) Syndrome of inappropriate secretion of antidiuretic hormone with exacerbated psychosis. Annals of Internal Medicine. 79. 551-554.

Fuint, A. J., Crosby, J. \& GenIK, J. L. (1996) Recurrent hyponatraemia associated with fluoxetine and paroxetine. American Journal of Psychiatry, 163, 134.

Glusac, E., PATel, H., Josef, N. C., et al (1990) Polydipsia and hyponatraemia induced by multiple neuroleptics but not molindone. Canadian Joumal of Psychiatry, 36. 268-269.

GODDARD, C. \& PATON, C. (1992) Hyponatraemia assoclated with paroxetine. Brttish Medical Joumal, s06. 1332. 
Jackson, C., Carson, W., Markowtr, J., et al (1995) SIADH associated with fluoxetine and sertraline therapy. American Journal of Psychiatry. 152, 809-810.

LEADBETTER, R. A. \& SHUTTY. M. S. (1994) Differential effects of neuroleptics and clozapine on polydipsia and intermittent hyponatraemia. Joumal of Clinical Psychiatry. 65[Suppl B], 110-113.

OGnve. A. D. \& Croy, M. F. (1992) Clozapine and hyponatraemia. Lancet, $\mathbf{3 4 0}, 672$.

OHSAWA. H., Kishimoto, T., HiRA. M., et al (1992) An epidemiological study on hyponatraemia in psychiatric patients in mental hospitals in Nara Pretecture. Japanese Journal of Psychiatry and Neurology. 46. 883-889.

RUTSKY, E. A. (1992) Water, electrolyte, mineral and acidbase metabolism. In Merk Manual of Diagnosis and Therapy (eds R. B. Berkow \& A. J. Fletcher), pp. 991995. New Jersey: Merk \& Co. Inc.

SCHWARTZ, W., BENNETt, S., CuRELOP, S., et al (1957) A syndrome of renal sodium loss and hyponatraemia probably resulting from inappropriate secretion of antidiuretic hormone. American Journal of Medicine, 23. 529-542.
StaAB, Y. P., Yerkes, S. A., Cheney, E. M., et al (1990) Transient SIADH associated with fluoxetine therapy. American Journal of Psychiatry, 147, 1569-1570.

Thomas. A. \& Verbaus. J. G. (1995) Hyponatraemia and the syndrome of inapproprlate antidiuretic hormone secretion associated with drug therapy in psychiatric patients. CNS Drugs, 4, 357-369.

THORNTON, S. L. \& RESCH. D. S. (1995) SIADH associated with sertraline therapy. American Journal of Psychiatry. 152. 809.

Vishwanath, B. M. Navalgund, A. A. Cusano, W. et al (1991) Fluoxetine as a cause of SIADH. American Joumal of Psychiatry, 148, 542-543.

WAKEFIELD, T. \& Cous, I. (1996). Clozapine treatment of a schizophrenic patient with polydipsia and hyponatraemia. Amerioan Journal of Psychiatry, 153, 445-446.

Robyn McAskill; and 'David Taylor, Chief Pharmacist, Maudsley Hospital, Denmark Hill, London SE5 8AZ

*Correspondence 